Research Article

\title{
Evolutionary Game Analysis of Information Sharing in Fresh Product Supply Chain
}

\author{
Yanhui Li (D, He Xu iD, and Yan Zhao iD \\ School of Information Management, Central China Normal University, Wuhan 430079, China \\ Correspondence should be addressed to Yan Zhao; yanz@mails.ccnu.edu.cn
}

Received 19 November 2020; Revised 4 February 2021; Accepted 27 February 2021; Published 8 March 2021

Academic Editor: Tingsong Wang

Copyright (c) 2021 Yanhui Li et al. This is an open access article distributed under the Creative Commons Attribution License, which permits unrestricted use, distribution, and reproduction in any medium, provided the original work is properly cited.

Fresh produce has increasingly become an important part of people's diet. However, the loss of fresh produce in the supply chain has existed for a long time and is difficult to overcome. Some companies use their own information management systems or use information systems built by other companies to release and manage fresh agricultural product information in a timely manner, thereby reducing product loss caused by the "bullwhip effect." However, this will also bring pressure on construction costs and the risk of information leakage. Based on the evolutionary game model, this paper conducts process modeling and analysis on the behavior of enterprise groups participating in information sharing. It is concluded that the greater the difference between the income obtained through information sharing and the cost of building information system, the higher the likelihood of enterprises participating in information sharing. In addition, the greater the profit from the construction of information platform, the smaller the profit of "free rider," and the smaller the risk of information leakage, the greater the enthusiasm of enterprises to participate in information sharing. Finally, some suggestions are proposed from the perspective of maximizing supply chain benefits.

\section{Introduction}

Fresh produce refers to the primary products that are sold without deep processing such as cooking and making but need to be kept fresh and simply arranged on the shelves. Fresh produce generally needs to be refrigerated and frozen to keep fresh, and bulk commodities need to be sold by weighing and barcoding, with a relatively short shelf life. Fresh produce mainly includes fruits, vegetables, meat, and aquatic products. With the improvement of people's living standards, fresh produce has become an important part of people's diet. However, the loss of fresh produce in the supply chain has existed for a long time and is difficult to overcome. In 2019, the transaction volume of China's fresh food market reached 2.04 trillion, with a year-on-year increase of $6.8 \%$. However, about $20 \%-30 \%$ of the fresh produce is wasted during different stages in the fresh product supply chain [1]. Compared with other commodities, such as daily necessities and household electrical appliances, the information asymmetry existing in the upstream and downstream of the fresh product supply chain has exacerbated the loss [2]. Therefore, information sharing behavior has a more profound impact on the business decisions of upstream suppliers and downstream retailers in the fresh product supply chain. Through information sharing, upstream and downstream companies can arrange production, sales, and inventory plans based on the shared information, which not only reduces operating costs of the supply chain but also is more flexible to market response; furthermore, it can reduce fresh produce loss of quantity and quality. According to Porter's competitive strategy theory [3], in order to gain advantages in negotiations, companies usually retain some private information. In the process of information sharing, information platform costs and risks of information leakage must also be considered. Once a company shares valuable private information with other companies, the company's control over the spread of this information in the supply chain will inevitably be weakened [4].

Therefore, how companies participate in the construction of information platforms and what form they use to share information has become a question worth exploring. 
From the perspective of bounded rationality, this paper uses evolutionary game theory to study the dynamic evolution of information sharing between upstream suppliers and downstream retailers in the fresh product supply chain and discusses the evolution law of enterprise group information sharing strategy in supply chain.

\section{Literature Review}

Due to the special deterioration properties of fresh produce, the research on the supply chain of fresh produce is usually classified into the research on the supply chain of deteriorated products. In 1913, in the inventory control model based on the classical economic order quantity (EOQ) model proposed by Harris, the implicit assumption is that the inventory goods have an infinite shelf life and will not deteriorate or damage [5]. As early as 1953, Abad believed that fashion goods would deteriorate after the prescribed storage period [6]. Almost ten years later, Ghare and Schrader first proposed the use of a negative exponential decay function to simulate deteriorating product inventory [7]. About 50 years later, based on different assumptions, many researchers have discussed different issues about the supply chain with deterioration. Hau et al. believe that the information transmitted in the form of "orders" is easily distorted and may mislead upstream members' inventory and production decisions. In particular, the variance of orders may be greater than the variance of sales. From downstream to upstream, distortion will also increase. And they named this phenomenon "bullwhip effect" [8].

Some scholars have studied the factors that influence corporate information sharing behavior. Cachon and Fisher found that traditional inventory management and information exchange technologies enable companies to quickly exchange information with companies on the information chain. The author compared the traditional strategy of not using shared information with the complete information strategy of using shared information. The supply chain cost under the complete information strategy is on average $2.2 \%$ lower than that of the traditional information strategy, and the maximum difference is $12.1 \%$. The authors believed that the implementation of information technology to accelerate and smooth the actual flow of goods in the supply chain is more valuable than the use of information technology to expand the flow of information [9]. Additionally, Huiping focused on analyzing the value created by information sharing, which reduced inventory levels in the three-level supply chain system. He discussed the coordination mechanism that upstream partners provide incentives for retailers through sharing profits and information sharing.

Some papers have explored the factors and mechanisms affecting information sharing. The author used the method of cooperative game to study the conflict of interest between the whole supply chain and a single partner and, finally, proposed a solution to how to effectively motivate partners to cooperate with each other to resolve the profit distribution between partners [10]. Luai et al. adopted a two-stage qualitative method. They conducted 40 interviews ( 4 in each case) through interviews with 7 experts and 10 comparative case studies on the relationship between producers and exporters. Supplemented by archival materials and nonparticipant observation, the author thought that specific affairs, relationships, and network drivers support information sharing in these relationships. This information sharing is linked to export performance. In the case of high performance, information sharing is triggered by integration-centric driving factors, and the focus is on long-term and joint planning on the basis of broader information sharing. In the case of poor performance, information sharing is triggered by more personalized driving factors [11]. Ye et al. [12] used CVaR criteria to measure risk and pointed out the influencing factors of demand information sharing from three different levels. Guo et al. studied the strategic information sharing behavior in two competitive channels: a decentralized supply chain and an integrated supply chain. The author believes that when competition in the retail market intensifies, retailers should reduce information sharing, but when their ability to obtain information improves, more information should be disclosed [13].

Many scholars have also used game theory to discuss the information sharing behavior in supply chain. In view of the asymmetry of supply and demand information between manufacturers and sellers, Wang Ying established a game model of information sharing, analyzed the changes in expected profits before and after sharing demand information and cost information, and eventually proposed an incentive mechanism for information sharing between manufacturers and distributors [14]. Esmaeili and Zeephongsekul proposed several seller-buyer supply chain models under the asymmetric information model and used the non-cooperative Stackelberg game model to study the incentive strategies when the buyer conceals the demand information and the seller conceals the cost information. The author also proposes a semi-cooperative model, which uses the sharing marketing expenditure as an incentive strategy to reveal information [15]. In traditional game theory, participants often participate in the game under complete information conditions, and they are often assumed to be completely rational. However, the complexity of the economic environment and the game problem itself causes incomplete information, which leads to the participants bounded rationally. Smith and Price have made contributions to the development of evolutionary game theory. Their evolutionary stability strategy has become the basic concept of evolutionary game theory [16, 17]. Furthermore, Bach, Helvikc, and Christiansen studied the divergence problem of the evolutionary stability strategy [18] and conducted related research on the cooperative evolutionary game problem in the case of one-party imitating [19].

In summary, to overcome the difficulties in information asymmetry in the supply chain and improve the level of information sharing, many studies have explored from the perspectives of technology applications, operation models, coordination mechanisms, incentive contracts, and competition forms. Different from supply chain of industrial products, the supply chain of fresh produce has the characteristics of short product cycle, volatile prices related to freshness, supply volume with a certain degree of 
randomness, and more uncontrollable factors. Because of the particularity of its products, the "bullwhip effect" in the supply chain has a more profound impact on the supply chain. Since the evolutionary game theory is aimed at lowrational parties that only have simple imitating ability, the decision-making method is to follow the crowd and is greatly affected by random factors. In addition, most of the fresh product supply chain companies are small- and medium-sized enterprises. When making information sharing decisions, they often refer to the decision-making behavior of companies of the same type. Although evolutionary game has been used in many fields, it is seldom used in the study of information sharing behavior involving fresh supply chain. Based on the existing research and the evolutionary game model, this paper conducts a study on the behavior of information sharing in the fresh product supply chain.

\section{Problem Description and Formulation}

In this section, we discuss the information sharing behavior between upstream suppliers and downstream retailers in a two-level supply chain of fresh produce. In the process of information sharing, the upstream and downstream enterprises attach great importance to measure the profit and the loss. The profit and loss brought by the information sharing behavior will reverse affect the information sharing decision of the upstream and downstream enterprises.

The basic idea of evolutionary game is as follows: in the game group with a certain scale, the players carry out repeated game activities. Due to bounded rationality, it is impossible for players to find the optimal equilibrium point in every game. They will focus on the successful players to improve their game behavior. Therefore, their best strategy is to imitate and improve the most advantageous strategy of the past. The object of evolutionary game theory is a "population," which focuses on the change in population structure, rather than the effect analysis of individual behavior. When all participants in a system adopt the "evolutionary stability strategy," the individuals who adopt other strategies will not be able to invade the system. Or, individuals who adopt other strategies will change their strategies or exit the system under the pressure of natural selection. That is to say, the information sharing behavior of the upstream and downstream enterprises in the fresh product supply chain will eventually reach a stable state, and the income will be higher than that of the enterprises adopting other strategies, so as to reach a stable level.

In the process of this game, the behavior of upstream and downstream enterprises is not completely rational, and the strategic combination of both sides is \{sharing, not sharing\}. Moreover, in order to meet the needs of consumers and obtain profits, the relationship between the two sides is fixed. In the process of game, one member is chosen from each group. Each member will adjust his strategy according to the choice of other members. The assumptions and related variables are as follows.

Assumption 1. Regarding the two-level supply chain system of the upstream and downstream enterprises of agricultural products supply chain as a dynamic system, both sides of the cooperation are bounded rationality, and they pursue the maximization of interests and have learning ability and speculation behavior. In the process of learning and imitating, both sides of the game seek better strategies through trial and error and selection, so as to achieve a dynamic balance.

Assumption 2. When upstream suppliers and downstream retailers share information, the "bullwhip effect" will be eliminated to a certain extent, and then both sides will have information sharing benefits from responding flexibly to market and reducing fresh produce loss. When sharing unilaterally, the other side will get additional benefits, while the sharing party will have potential losses due to the risk of information leakage in addition to benefits and construction costs.

Assumption 3. Upstream supplier A (downstream retailer A) can make use of the information platform unilaterally constructed by downstream retailer B (upstream supplier b) and make use of the other party's information platform in a certain extent through "free rider," so as to obtain certain benefits. However, because the information platform is built according to the needs of enterprise B (upstream supplier B), the profit obtained by enterprise $B$ through the construction of information platform is more than that of enterprise a by " free rider" $(\lambda>\beta)$.

For ease of reference, the list of notations is shown in Table 1.

Based on the above assumptions, we can establish the payoff matrix $U$ of both sides of the game.

\section{Evolutionary Game Analysis}

4.1. Equilibrium Analysis. According to the above payment matrix, the game is analyzed and solved by the bounded rationality game model.

According to Table 2, the expected payoff for upstream supplier sharing information is

$$
\begin{aligned}
u_{11} & =y\left(\pi_{1}+\beta_{1} l-\theta_{1}-\alpha C\right)+(1-y) \pi_{1}+\beta_{1} l-\theta_{1}-C \\
& =\pi_{1}+\beta_{1} l-\theta_{1}-C+(1-\alpha) y C .
\end{aligned}
$$

The expected payoff for upstream supplier not sharing information is

$$
u_{12}=y\left(\pi_{1}+\lambda_{1} l\right)+(1-y) \pi_{1}=\pi_{1}+y \lambda_{1} l .
$$

The average expected payoff for upstream supplier is

$$
\begin{aligned}
u_{1}= & x u_{11}+(1-x) u_{12}=x\left[\pi_{1}+\beta_{1} l-\theta_{1}-C+(1-\alpha) y C\right] \\
& +(1-x)\left(\pi_{1}+y \lambda_{1} l\right)=\pi_{1}+y \lambda_{1} l \\
& +x\left[\pi_{1}+\beta_{1} l-\theta_{1}-C+(1-\alpha) y C-\pi_{1}-y \lambda_{1} l\right] .
\end{aligned}
$$

The replicator dynamics equation of upstream supplier is 
TABLE 1: List of notations.

\begin{tabular}{|c|c|}
\hline Symbol & Description \\
\hline$x$ & The proportion of upstream suppliers participating in information of fresh product sharing \\
\hline $1-x$ & The proportion of upstream suppliers not participating in information of fresh product sharing \\
\hline$y$ & The proportion of downstream retailers participating in information of fresh product sharing \\
\hline & The proportion of downstream retailers not participating in information of fresh product sharing \\
\hline & The additional revenue of two-echelon supply chain due to information sharing \\
\hline C & The information of fresh product sharing cost \\
\hline$\pi_{1}$ & The upstream supplier's income, when the information is not shared \\
\hline$\pi_{2}$ & The downstream retailer's income, when the information is not shared \\
\hline$\beta_{1}$ & $\begin{array}{c}\text { The percentage of the additional revenue of upstream supplier in the additional revenue of whole supply chain due to } \\
\text { participation in information sharing }\end{array}$ \\
\hline$\beta_{2}$ & $\begin{array}{c}\text { The percentage of the additional revenue of downstream retailer in the additional revenue of whole supply chain due to } \\
\text { participation in information sharing }\end{array}$ \\
\hline$\lambda_{1}$ & $\begin{array}{c}\text { The percentage of the additional revenue of upstream supplier in the additional revenue of whole supply chain due to unilateral } \\
\text { use of shared information from downstream retailer }\end{array}$ \\
\hline$\lambda_{2}$ & $\begin{array}{c}\text { The percentage of the additional revenue of downstream retailer in the additional revenue of whole supply chain due to unilateral } \\
\text { use of shared information from upstream supplier }\end{array}$ \\
\hline$\theta_{1}$ & The loss of information leakage caused by upstream supplier sharing information \\
\hline$\theta_{2}$ & The loss of information leakage caused by downstream retailer sharing information \\
\hline$\alpha$ & $\begin{array}{c}\text { The proportion of the information sharing cost borne by the upstream supplier, when the supply chain participants are all } \\
\text { involved in information sharing }\end{array}$ \\
\hline $1-\alpha$ & $\begin{array}{c}\text { The proportion of the information sharing cost borne by the downstream retailer, when the supply chain participants are all } \\
\text { involved in information sharing }\end{array}$ \\
\hline
\end{tabular}

TABLE 2: Information sharing evolutionary game payoff matrix.

\begin{tabular}{|c|c|c|c|}
\hline & & \multicolumn{2}{|c|}{ Downstream retailer } \\
\hline & & Sharing $(y)$ & Not sharing $(1-y)$ \\
\hline Upstream supplier & $\begin{array}{c}\text { Sharing }(x) \\
\text { Not sharing }(1-x)\end{array}$ & $\begin{array}{c}\pi_{1}+\beta_{1} l-\theta_{1}-\alpha C \\
\pi_{2}+\beta_{2} l-\theta_{2}-(1-\alpha) C \\
\pi_{1}+\lambda_{1} l \\
\pi_{2}+\beta_{2} l-\theta_{2}-C\end{array}$ & $\begin{array}{c}\pi_{1}+\beta_{1} l-\theta_{1}-C \\
\pi_{2}+\lambda_{2} l \\
\pi_{1}, \pi_{2}\end{array}$ \\
\hline
\end{tabular}

$$
\begin{aligned}
F(x) & =\frac{\mathrm{d} x(t)}{t}=x\left(u_{11}-u_{1}\right)=x(1-x)\left(u_{11}-u_{12}\right) \\
& =x(1-x)\left[\beta_{1} l-\theta_{1}-C+(1-\alpha) y C-y \lambda_{1} l\right] .
\end{aligned}
$$

The expected payoff for downstream retailer sharing information is

$$
\begin{aligned}
u_{21}= & x\left[\pi_{2}+\beta_{2} l-\theta_{2}-(1-\alpha) C\right]+(1-x)\left(\pi_{2}+\beta_{2} l\right. \\
& \left.-\theta_{2}-C\right)=\pi_{2}+\beta_{2} l-\theta_{2}-C+x \alpha C .
\end{aligned}
$$

The expected payoff for downstream retailer not sharing information is

$$
u_{22}=x\left(\pi_{2}+\lambda_{2} l\right)+(1-x) \pi_{2}=\pi_{2}+x \lambda_{2} l .
$$

The average expected payoff for downstream retailer is

$$
\begin{aligned}
u_{2} & =y u_{21}+(1-y) \\
u_{22} & =y\left(\pi_{2}+\beta_{2} l-\theta_{2}-C+x \alpha C\right)+(1-y)\left(\pi_{2}+x \lambda_{2} l\right) \\
& =\pi_{2}+x \lambda_{2} l+y\left(\pi_{2}+\beta_{2} l-\theta_{2}-C+x \alpha C-\pi_{2}-x \lambda_{2} l\right) \\
& =\pi_{2}+x \lambda_{2} l+y\left(\beta_{2} l-\theta_{2}-C+x \alpha C-x \lambda_{2} l\right) .
\end{aligned}
$$

is

The replicator dynamics equation of downstream retailer

$$
\begin{aligned}
F(y) & =\frac{\mathrm{d} y(t)}{t}=y\left(u_{11}-u_{1}\right)=y(1-y)\left(u_{21}-u_{22}\right) \\
& =y(1-y)\left(\beta_{2} l-\theta_{2}-C+x \alpha C-x \lambda_{2} l\right) .
\end{aligned}
$$

Let $F(x)=0$ and $F(y)=0$, then five singularities can be gained:

$O=(0,0), A=(1,0), B=(0,1), C=(1,1)$, and $D=$ $\left(x^{*}, y^{*}\right)$, where $x^{*}=\left(C+\theta_{2}-\beta_{2} l / \alpha C-\lambda_{2} l\right)$ and $y^{*}=$ $\left(C+\theta_{1}-\beta_{1} l /(1-\alpha) C-\lambda_{1} l\right)$. By solving $F^{\prime}(x)$ and $F_{\prime}^{\prime}(y)$ separately, we have $F^{\prime}(x)=(1-2 x)\left[\beta_{1} l-\theta_{1}-C+(1-\alpha)\right.$ $\left.y C-y \lambda_{1} l\right] \quad$ and $\quad F^{\prime}(y)=(1-2 y)\left(\beta_{2} l-\theta_{2}-C+x \alpha C\right.$ $\left.-x \lambda_{2} l\right)$. Then, let $F^{\prime}(x)=0$ and $F^{\prime}(y)=0$, we can get the solutions: $x_{1}=(1 / 2)$ and $y_{1}=(1 / 2), x_{2}=\left(C+\theta_{2}-\beta_{2} l / \alpha C\right.$ $\left.-\lambda_{2} l\right)$, and $y_{2}=\left(C+\theta_{1}-\beta_{1} l /(1-\alpha) C-\lambda_{1} l\right)$.

From the above results, when $x^{*}$ and $y^{*}$ exist, we get $\alpha C>\lambda_{2} l,(1-\alpha) C>\lambda_{1} l, C+\theta_{2}>\beta_{2} l, C+\theta_{1}>\beta_{1} l$. The cost shared by all parties in the cooperative construction of information platform is greater than the benefits brought by "free rider." And the total construction cost is greater than the difference between the profit brought by information 
sharing minus the risk of information leakage when the two sides share information. The following is a detailed analysis. When

$\alpha C<\lambda_{2} l<\beta_{2} l-\theta_{2},(1-\alpha) C<\lambda_{1} l<\beta_{1} l-\theta_{1}, C<\beta_{2} l-$ $\theta_{2}, C<\beta_{1} l-\theta_{1}$, whether it is upstream or downstream, choosing information sharing is a dominant strategy. Choosing to share information always has more benefits than not sharing information. Then, the game will have the Nash equilibrium \{sharing, sharing\}. From this, we can also see that, with the development of information technology, when the cost of information construction is low to a certain extent, the two sides of the game will definitely choose to share information to maximize their own benefits.

4.2. Stability Analysis of Equilibrium. Use the local stability analysis of the Jacobian matrix to analyze the local stability of each point. The Jacobian matrix of the evolutionary game is $J=\left(\begin{array}{ll}((\mathrm{d} x / \mathrm{d} t) / \mathrm{d} x) & ((\mathrm{d} x / \mathrm{d} t) / \mathrm{d} y) \\ ((\mathrm{d} y / \mathrm{d} t) / \mathrm{d} x) & ((\mathrm{d} y / \mathrm{d} t) / \mathrm{d} y)\end{array}\right)$. It can be obtained by substitution:

$$
J=\left(\begin{array}{cc}
(1-2 x)\left[\beta_{1} l-\theta_{1}-C+(1-\alpha) y C-y \lambda_{1} l\right] & x(1-x)\left[(1-\alpha) C-\lambda_{1} l\right] \\
y(1-y)\left(\alpha C-\lambda_{2} l\right) & (1-2 y)\left(\beta_{2} l-\theta_{2}-C+x \alpha C-x \lambda_{2} l\right)
\end{array}\right),
$$

in which

$$
\begin{aligned}
\operatorname{det} J= & (1-2 x)\left[\beta_{1} l-\theta_{1}-C+(1-\alpha) y C-y \lambda_{1} l\right] *(1-2 y)\left(\beta_{2} l-\theta_{2}-C+x \alpha C-x \lambda_{2} l\right)-x(1-x)\left[(1-\alpha) C-\lambda_{1} l\right] \\
& * y(1-y)\left(\alpha C-\lambda_{2} l\right), \\
\operatorname{tr} J= & (1-2 x)\left[\beta_{1} l-\theta_{1}-C+(1-\alpha) y C-y \lambda_{1} l\right]+(1-2 y)\left(\beta_{2} l-\theta_{2}-C+x \alpha C-x \lambda_{2} l\right) .
\end{aligned}
$$

The equilibrium point satisfying the determinant $\operatorname{det} J>0$ and the trace of matrix $\operatorname{tr} J<0$ is the evolutionary stability strategy of the system. Calculating the expressions of determinant det $J$ and trace $\operatorname{tr} J$ of the matrix at five equilibrium points, the results are shown in Table 3.

According to the above expression, the stability of the equilibrium point of the game is analyzed. The results are shown in Table 4.

4.3. Analysis of Evolutionary Game Results. According to the stability theorem of replicator equation and the properties of evolutionary stable strategy, when $F(y)=0, F^{\prime}(y)<0, y^{*}$ is an evolutionary stable strategy. The following is discussion.

(1) When $x^{*}<\left(C+\theta_{2}-\beta_{2} l / \alpha C-\lambda_{2} l\right)$, for $y^{*}=0$, $F\left(y^{*}\right)=0, \quad F^{\prime}\left(y^{*}\right)<0, \quad y^{*}=0$ is asymptotically stable points. When the number of upstream suppliers participating in information sharing does not reach a certain amount or is relatively small, the proportion of downstream suppliers sharing information decreases. The changing trend of imitator dynamic evolution is shown in Figure 1.

(2) When $x^{*}=\left(C+\theta_{2}-\beta_{2} l / \alpha C-\lambda_{2} l\right)$, for $F(y)=0$, $F^{\prime}(y)=0$, all the values of the $y$ are in a stable state. If the proportion of the upstream suppliers participating in information sharing reaches $\left(C+\theta_{2}-\beta_{2} l / \alpha C-\lambda_{2} l\right)$, the dynamic evolution state of downstream retailers is relatively stable.

(3) When $x^{*}>\left(C+\theta_{2}-\beta_{2} l / \alpha C-\lambda_{2} l\right)$, for $y^{*}=1$, $F\left(y^{*}\right)=0, \quad F^{\prime}\left(y^{*}\right)<0, \quad y^{*}=1$ is asymptotically stable points. When the number of upstream suppliers participating in information sharing reaches a certain amount or relatively large, the proportion of downstream suppliers sharing information increases.

Same as above, when $F(x)=0, F^{\prime}(x)<0, y^{*}$ is an evolutionary stable strategy. The following is discussion.

(1) When $y^{*}<\left(\left(C+\theta_{1}-\beta_{1} l\right) /\left((1-\alpha) C-\lambda_{1} l\right)\right)$, for $x^{*}=0, F\left(x^{*}\right)=0, F^{\prime}\left(x^{*}\right)<0, y^{*}=0$ is asymptotically stable points. When the number of downstream retailers participating in information sharing does not reach a certain amount or is relatively small, the proportion of upstream suppliers sharing information decreases. The changing trend of imitator dynamic evolution is shown in Figure 2.

(2) When $y^{*}=\left(\left(C+\theta_{1}-\beta_{1} l\right) /\left((1-\alpha) C-\lambda_{1} l\right)\right)$, for $F(x)=0, F^{\prime}(x)=0$, all the values of $x$ are in a stable state. If the proportion of the downstream retailers participating in information sharing reaches $\left(\left(C+\theta_{1}-\beta_{1} l\right) /\left((1-\alpha) C-\lambda_{1} l\right)\right)$, the dynamic evolution state of upstream suppliers is relatively stable.

(3) When $y^{*}>\left(\left(C+\theta_{1}-\beta_{1} l\right) /\left((1-\alpha) C-\lambda_{1} l\right)\right)$, for $y^{*}=1, F\left(y^{*}\right)=0, F^{\prime}\left(y^{*}\right)<0, y^{*}=1$ is asymptotically stable points. When the number of downstream retailers participating in information sharing reaches a certain amount or is relatively large, the proportion of upstream suppliers sharing information increases.

As discussed above, if the cost is small enough, there will always be more additional benefits from sharing, and all companies will choose to share information. When 
TABle 3: The determinant $\operatorname{det} J$ and $\operatorname{trace} \operatorname{tr} J$ of equilibrium point.

\begin{tabular}{lcc}
\hline Equilibrium point & Determinant det $J$ & $\operatorname{Trace} \operatorname{tr} J$ \\
\hline$(0,0)$ & $\left(\beta_{2} l-\theta_{2}-C\right) *\left(\beta_{1} l-\theta_{1}-C\right)$ & $\left(\beta_{2} l-\theta_{2}-C\right)+\left(\beta_{1} l-\theta_{1}-C\right)$ \\
$(0,1)$ & $\left(\beta_{1} l-\theta_{1}-\alpha C-\lambda_{1} l\right) *(-)\left(\beta_{2} l-\theta_{2}-C\right)$ & {$\left[\beta_{1} l-\theta_{1}-\alpha C-\lambda_{1} l\right]-\left(\beta_{2} l-\theta_{2}-C\right)$} \\
$(1,0)$ & $-\left(\beta_{1} l-\theta_{1}-C\right) *\left(\beta_{2} l-\theta_{2}-(1-\alpha) C-\lambda_{2} l\right)$ & $-\left(\beta_{1} l-\theta_{1}-C\right)+\left(\beta_{2} l-\theta_{2}-(1-\alpha) C-\lambda_{2} l\right)$ \\
$(1,1)$ & $\left(\beta_{1} l-\theta_{1}-\alpha C-\lambda_{1} l\right) *\left[\beta_{2} l-\theta_{2}-(1-\alpha) C-\lambda_{2} l\right]$ & $-\left(\beta_{1} l-\theta_{1}-\alpha C-\lambda_{1} l\right)-\left[\beta_{2} l-\theta_{2}-(1-\alpha) C-\lambda_{2} l\right]$ \\
$\left(x^{*}, y^{*}\right)$ & $-x^{*}\left(1-x^{*}\right)\left[(1-\alpha) C-\lambda_{1} l\right] * y^{*}\left(1-y^{*}\right)\left(\alpha C-\lambda_{2} l\right)$ & 0 \\
\hline
\end{tabular}

TABle 4: Stability analysis of equilibrium point.

\begin{tabular}{|c|c|c|c|c|}
\hline Known conditions & Equilibrium point & Symbol of determinant & Symbol of trace & Result \\
\hline Condition 1 & $O(0,0)$ & + & - & ESS \\
\hline$\beta_{1} l-\theta_{1}-C<0$ & $A(0,1)$ & - & Uncertain & Unstable \\
\hline$\beta_{2} l-\theta_{2}-C<0$ & $B(1,0)$ & - & Uncertain & Unstable \\
\hline$\beta_{1} l-\theta_{1}-\alpha C-\lambda_{1} l<0$ & $C(1,1)$ & + & + & Unstable \\
\hline$\beta_{2} l-\theta_{2}-(1-\alpha) C-\lambda_{2} l<0$ & $D\left(x^{*}, y^{*}\right)$ & - & 0 & Saddle poin \\
\hline Condition 2 & $O(0,0)$ & + & - & ESS \\
\hline$\beta_{1} l-\theta_{1}-C<0$ & $A(0,1)$ & + & + & Unstable \\
\hline$\beta_{2} l-\theta_{2}-C<0$ & $B(1,0)$ & - & Uncertain & Unstable \\
\hline$\beta_{1} l-\theta_{1}-\alpha C-\lambda_{1} l>0$ & $C(1,1)$ & - & Uncertain & Unstable \\
\hline$\beta_{2} l-\theta_{2}-(1-\alpha) C-\lambda_{2} l<0$ & $D\left(x^{*}, y^{*}\right)$ & - & 0 & Saddle poin \\
\hline Condition 3 & $O(0,0)$ & + & - & ESS \\
\hline$\beta_{1} l-\theta_{1}-C<0$ & $A(0,1)$ & - & Uncertain & Unstable \\
\hline$\beta_{2} l-\theta_{2}-C<0$ & $B(1,0)$ & + & + & Unstable \\
\hline$\beta_{1} l-\theta_{1}-\alpha C-\lambda_{1} l<0$ & $C(1,1)$ & - & Uncertain & Unstable \\
\hline$\beta_{2} l-\theta_{2}-(1-\alpha) C-\lambda_{2} l>0$ & $D\left(x^{*}, y^{*}\right)$ & - & 0 & Saddle poin \\
\hline Condition 4 & $O(0,0)$ & + & - & ESS \\
\hline$\beta_{1} l-\theta_{1}-C<0$ & $A(0,1)$ & - & Uncertain & Unstable \\
\hline$\beta_{2} l-\theta_{2}-C<0$ & $B(1,0)$ & - & Uncertain & Unstable \\
\hline$\beta_{1} l-\theta_{1}-\alpha C-\lambda_{1} l>0$ & $C(1,1)$ & + & - & ESS \\
\hline$\beta_{2} l-\theta_{2}-(1-\alpha) C-\lambda_{2} l>0$ & $D\left(x^{*}, y^{*}\right)$ & - & 0 & Saddle poin \\
\hline
\end{tabular}

$1>x^{*}>0$ and $1>y^{*}>0$, for $y=\left(\left(C+\theta_{1}-\beta_{1} l\right) /((1-\alpha)\right.$ $\left.\left.C-\lambda_{1} l\right)\right)$, equation (4) is always be equal to 0 , and $x$ is stable. For $y>\left(\left(C+\theta_{1}-\beta_{1} l\right) /\left((1-\alpha) C-\lambda_{1} l\right)\right)$, equation (4) is positive and $x=0, x=1$ is a two-stable stage, where $x=1$ is an evolutionary stable strategy. For $y<\left(\left(C+\theta_{1}-\beta_{1} l\right) /((1-\right.$ $\left.\alpha) C-\lambda_{1} l\right)$ ), equation (4) is negative, and $x=0, x=1$ is a two-stable stage, where $x=0$ is an evolutionary stable strategy. The evolutionary stable strategy of $y$ can be analyzed in the same way of $x$. The dynamic relationship between the above two groups is represented by a twodimensional drawing, as shown in Figure 3.

Point $\mathrm{O}$ indicates that both upstream and downstream enterprises do not share information, while point $\mathrm{C}$ indicates that both upstream and downstream enterprises participate in information sharing. When the profit of information sharing is greater than that of building information system, both sides will adopt information sharing strategy after longterm game. Through the formula derivation, we can observe that the basic income $\pi$ when information is not shared has no influence on the formation of decision equilibrium state.

With the improvement in people's living standards, the demand for fresh produce is also increasing. Information sharing in the supply chain of farm products is an effective means to improve the efficiency of the supply chain and reduce the consumption rate of fresh produce. This is also in line with the basic cognition. In fact, enterprises in fresh product supply chain may not be completely rational and often choose to follow others. Through the above model, it can be seen that the larger the $S_{\mathrm{ADBC}}$ area, the greater the possibility of enterprises to participate in information sharing. The area of $S_{\mathrm{ADBC}}$ is related to the difference in income from information sharing and cost of building information system. The greater the difference, the greater the probability of upstream and downstream enterprises participating in information sharing. We can observe that the closer point $\mathrm{D}$ is to point $\mathrm{O}$, the larger area of $S_{\mathrm{ADBC}}$ is, the more likely enterprises are to share information. That is, $\left(\left(C+\theta_{2}-\beta_{2} l\right) /\left(\alpha C-\lambda_{2} l\right)\right)$

$\left(\left(C+\theta_{1}-\beta_{1} l\right) /\left((1-\alpha) C-\lambda_{1} l\right)\right)$ the smaller, the greater the possibility of information sharing. We can infer that the smaller the "free rider" revenue ratio $(\lambda)$ is, the greater the proportion of construction income $(\beta)$ is, the less loss caused by information leakage and the greater the possibility of enterprise information sharing is. The following text will use numerical simulation to verify this.

\section{Numerical Simulation}

In order to elaborate the evolution game more intuitively, we make some numerical analyses to simulate the dynamic evolution of its strategy select in different situations. We take initial point at 0.1 intervals and set the time step to 1 . Referring to the research of Cai et al. [20-22], the parameter values are $\alpha=0.5, C=20$, and $l=100$. 


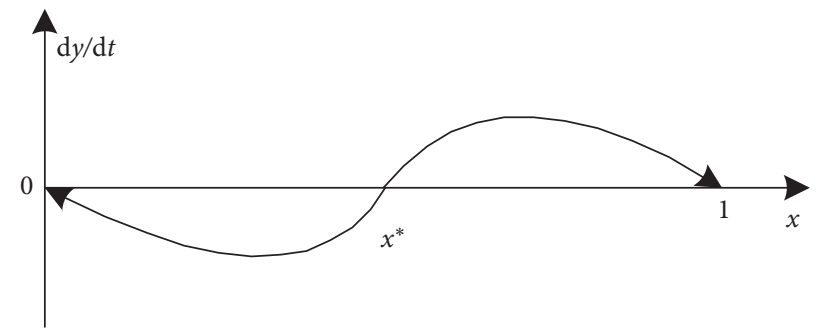

Figure 1: Changing trends of information sharing in evolutionary game $x^{*}=\left(\left(C+\theta_{2}-\beta_{2} l\right) /\left(\alpha C-\lambda_{2} l\right)\right)$.

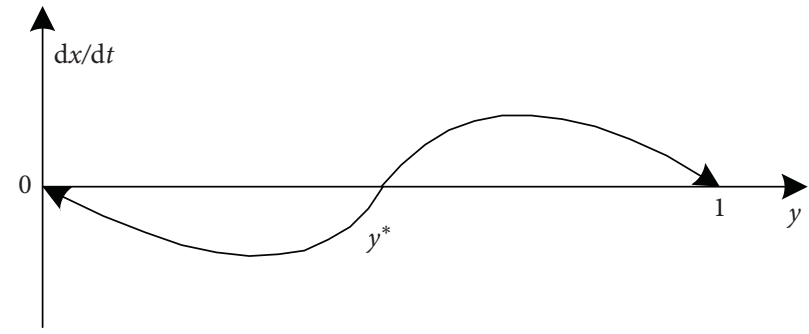

FIGURE 2: Changing trends of information sharing in evolutionary game $y^{*}=\left(\left(C+\theta_{1}-\beta_{1} l\right) /\left((1-\alpha) C-\lambda_{1} l\right)\right)$.

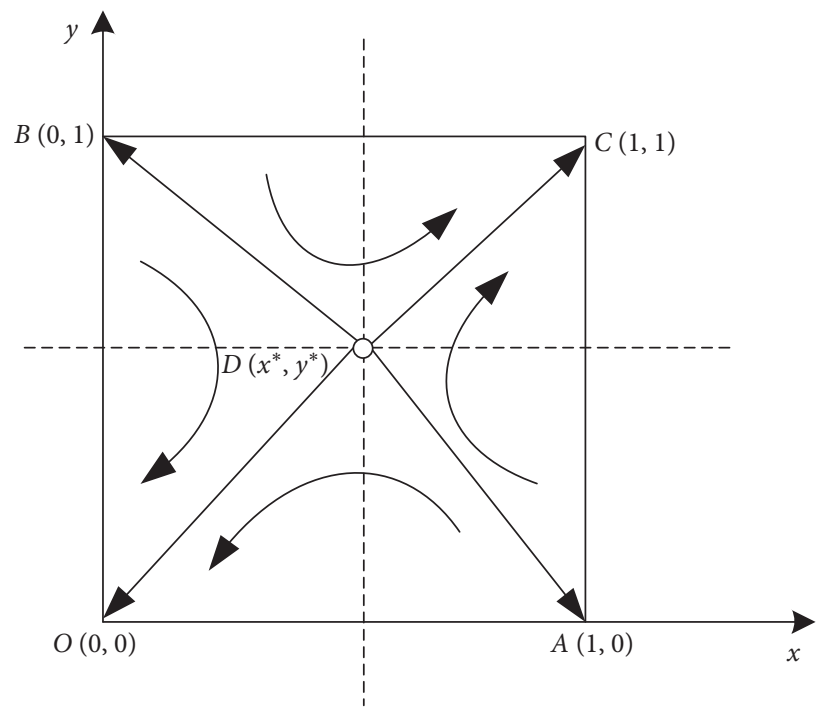

FIGURE 3: Schematic diagram of group replication dynamics and stability of both parties in the game.

(1) Assuming that $\lambda_{1}=\lambda_{2}=0.05, \theta_{1}=\theta_{2}=5$. The values of $\beta$ are assumed to be $0.1,0.2,0.3$, and 0.4 . In the case of different $\beta$, the dynamic evolution process of enterprise's strategy is shown in Figure 4 . When $\beta$ is larger, enterprises gradually change from unwilling to share to willing to share. Because enterprises benefit more from information sharing, when $\beta$ increases, the greater the percentage of the additional revenue $(\beta)$, the faster the enterprises participate in information sharing.

(2) Assuming that $\theta_{1}=\theta_{2}=5, \beta_{1}=\beta_{2}=0.3$. The values of $\lambda$ are assumed to be $0,0.05,0.075$, and 0.1 . In the case of different $\lambda$, the dynamic evolution process of enterprise's strategy is shown in Figure 5. The larger the percentage of the additional revenue $(\lambda)$ due to unilateral use of shared information from others, the slower the enterprises participating in information sharing.

(3) Assuming that $\beta_{1}=\beta_{2}=0.3, \lambda_{1}=\lambda_{2}=0.05$. The values of $\theta$ are assumed to be $0,5,10$, and 15 . In the case of different $\theta$, the dynamic evolution process of enterprise's strategy is shown in Figure 6. With the increase in information leakage loss, enterprises gradually change their strategies from information sharing to not sharing. 


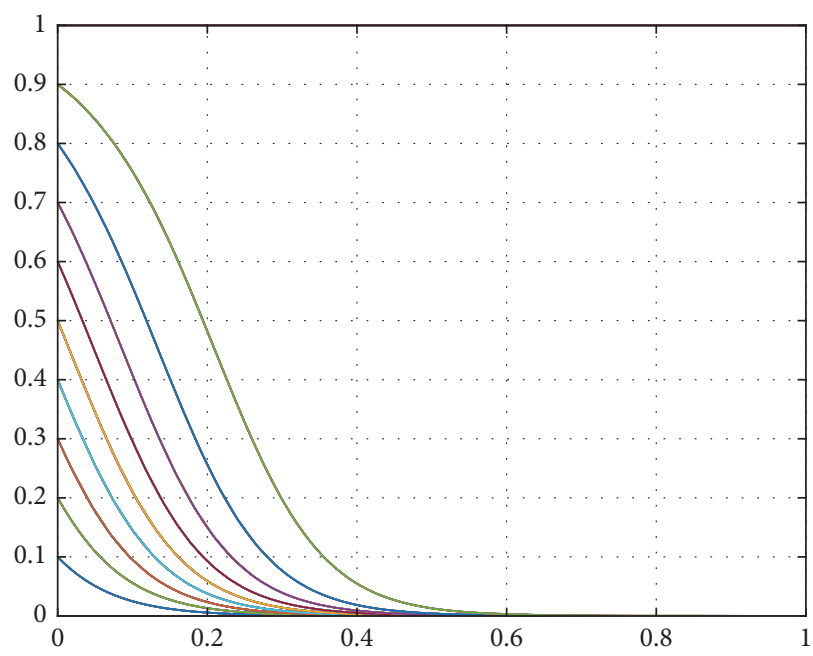

(a)

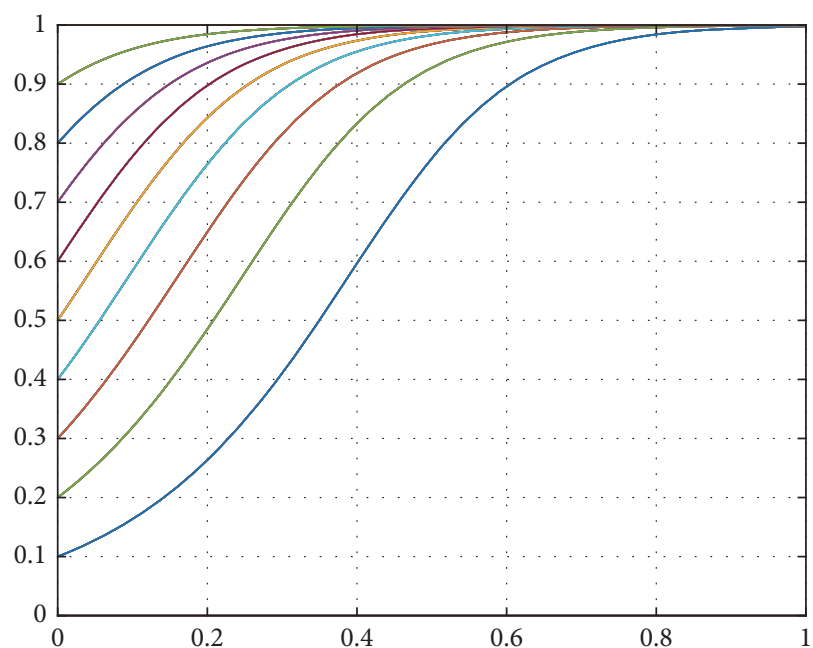

(c)

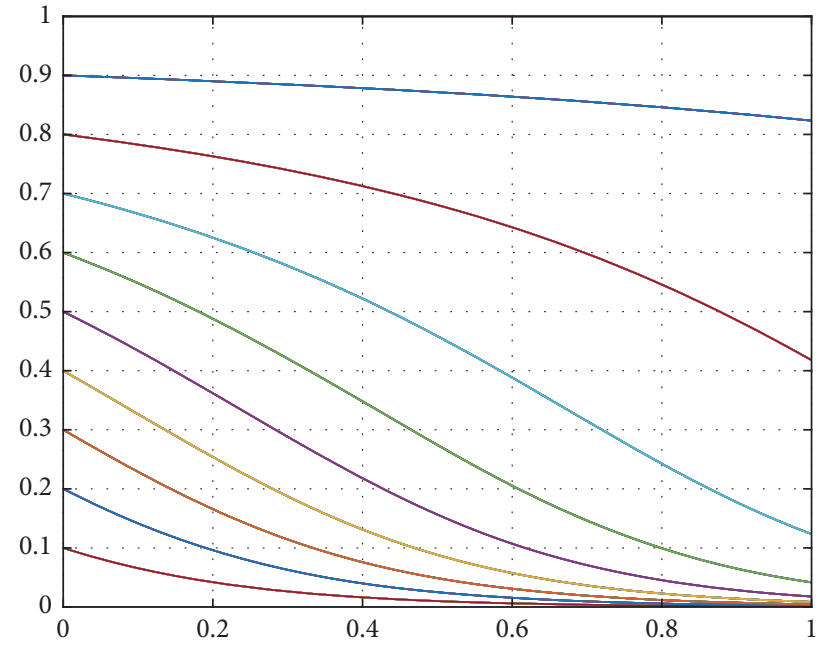

(b)

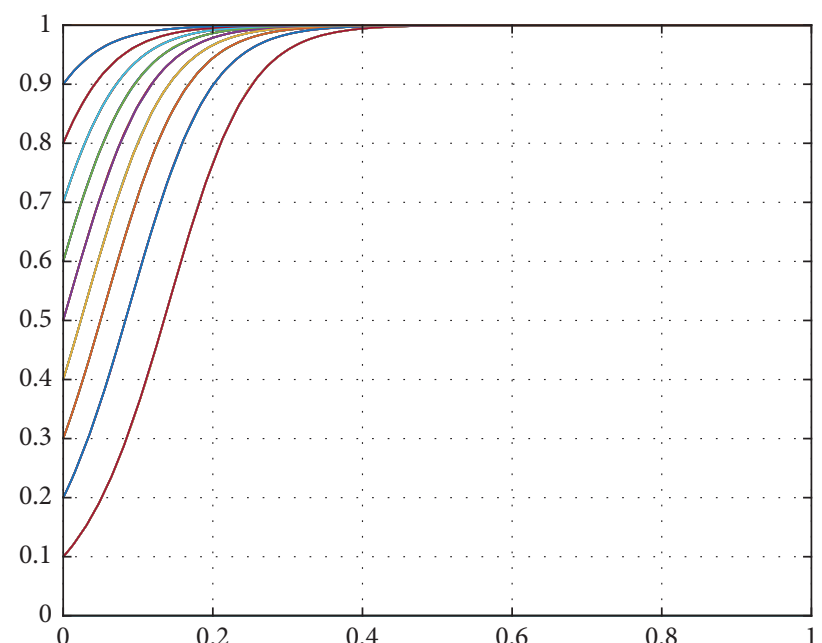

(d)

Figure 4: In the case of different $\beta$ values, dynamic evolution process of information sharing: (a) $\beta_{1}-\beta_{2}=0.1$; (b) $\beta_{1}-\beta_{2}=0.2$; (c) $\beta_{1}-\beta_{2}=0.3$; (d) $\beta_{1}-\beta_{2}=0.4$.

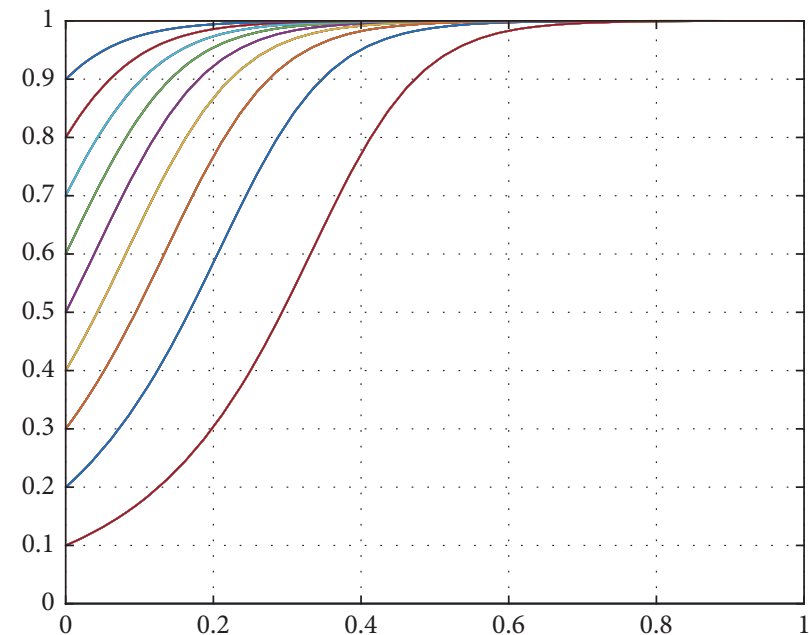

(a)

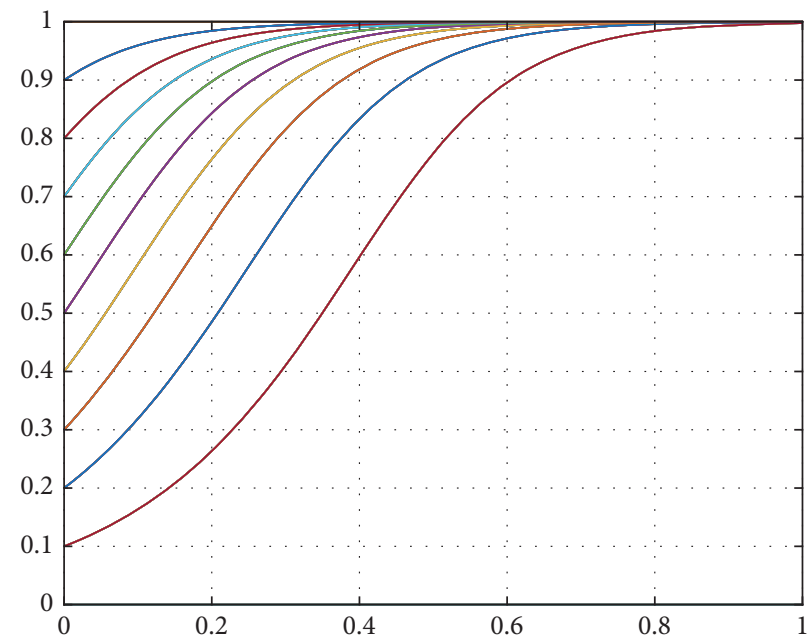

(b)

Figure 5: Continued. 


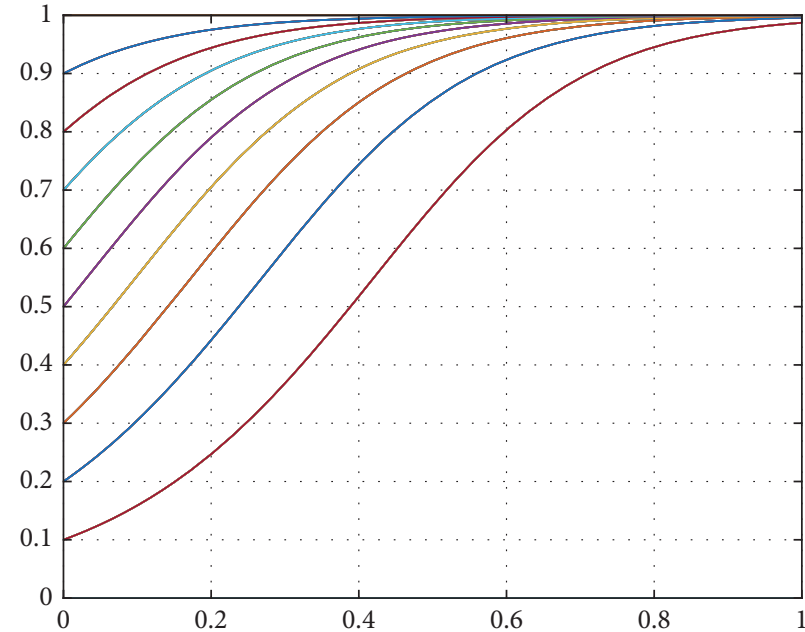

(c)

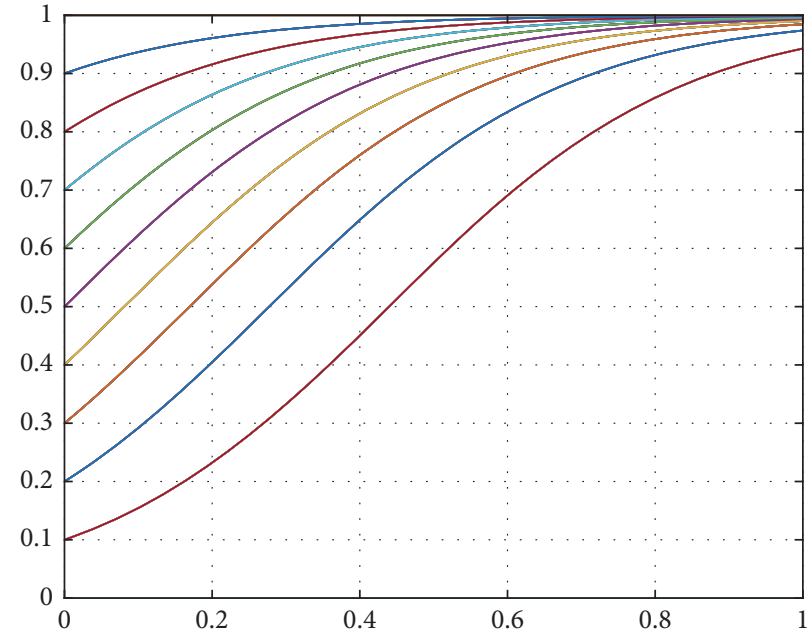

(d)

Figure 5: In the case of different $\lambda$ values, dynamic evolution process of information sharing: (a) $\lambda_{1}=\lambda_{2}=0 ;(\mathrm{b}) \lambda_{1}=\lambda_{2}=0.05$; (c) $\lambda_{1}=\lambda_{2}=0.075$; (d) $\lambda_{1}=\lambda_{2}=0.1$.

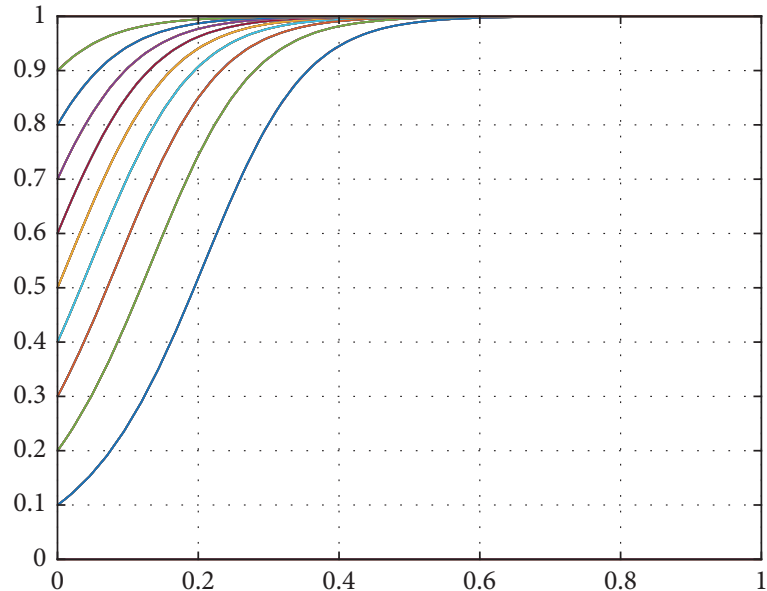

(a)

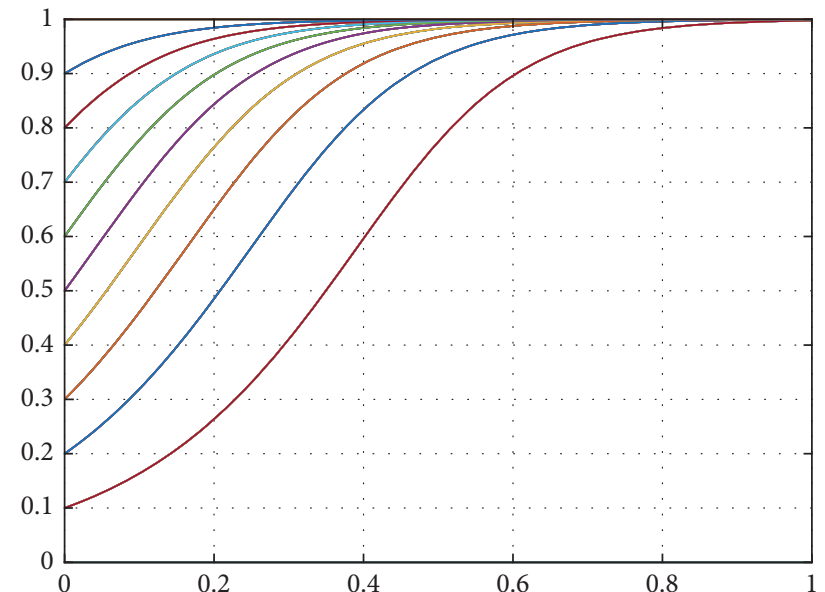

(b)

Figure 6: Continued.

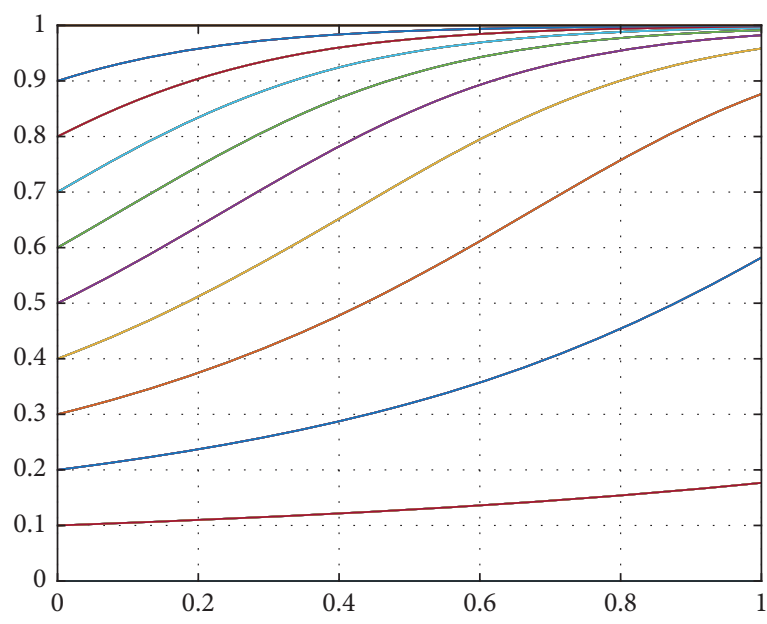

(c)

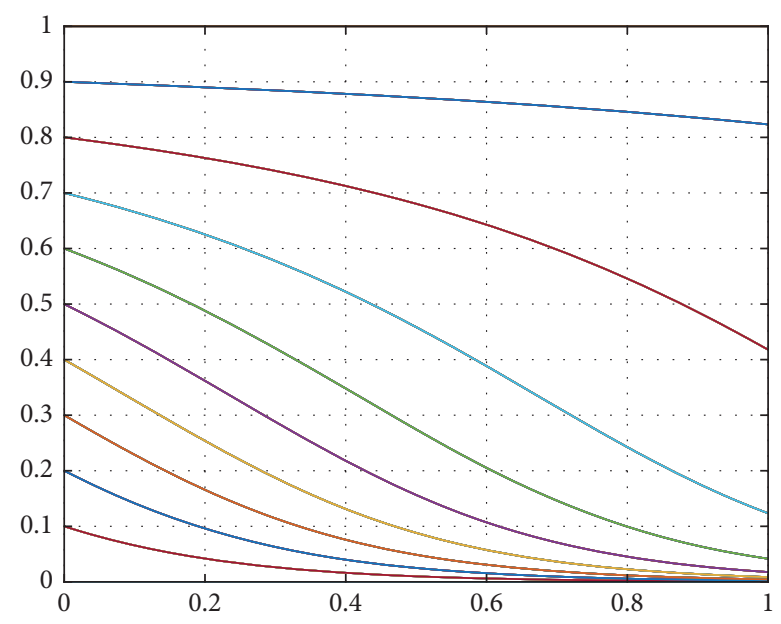

(d)

Figure 6: In the case of different $\theta$ values, dynamic evolution process of information sharing: (a) $\theta_{1}=\theta_{2}=0$; (b) $\theta_{1}=\theta_{2}=5$; (c) $\theta_{1}=\theta_{2}=10$; (d) $\theta_{1}=\theta_{2}=15$. 


\section{Conclusions}

Information sharing in the fresh product supply chain is an effective means to improve the flexibility of the supply chain and reduce the consumption of fresh produce. To solve the loss of fresh produce in a two-echelon supply chain, some companies build their own information management systems or use information systems built by other companies to share and manage the information of fresh produce in a timely manner, thereby reducing product wastage brought by the "bullwhip effect." In practical situation, companies may not be completely rational and often follow suit. Based on the evolutionary game and the characteristics of fresh produce, this paper conducts process modeling and analysis on the behavior of upstream suppliers and downstream retailers participating in information sharing. Some conclusions are drawn as follows:

(1) The larger the difference between the profit obtained through information sharing and the cost of building information system, the greater the probability of upstream suppliers and downstream retailers participating in information sharing.

(2) When the proportion of construction revenue $(\beta)$ is greater, "free rider" revenue ratio $(\lambda)$ is smaller, and the loss $(\theta)$ of information leakage caused by information sharing is smaller, upstream suppliers and downstream retailers are more likely to participate in information sharing.

Based on the above findings, some management implications can be got as follows:

(1) For upstream wholesalers and downstream retailers, the loss and cost can be reduced effectively by building information sharing platforms, such as ERP, OMS, WMS, TMS, and DMS. At the same time, in order to improve the profit of supply chain members from information sharing, the government can give incentives and subsidies to the construction of information platform and launch farmers' information technology training, to encourage supply chain members to participate in information sharing more actively. In this way, the quality and the timeliness of fresh produce will be improved, and ultimately the efficiency of the whole fresh product supply chain also will be improved.

(2) When the supply chain members use the information sharing system that has been built by the other party, the platform builder can charge the management fee of the information platform or providing the system interface with compensation. By reducing the "free rider" income of the members who do not share information, the progress of their participation in information sharing speeds up. Furthermore, the members can obtain a certain proportion of extraprofits brought by information sharing by means of incentive contracts such as the revenue sharing contract, which can also encourage those who do not share information to change their strategies to participate in information sharing. At the same time, the loss caused by information leakage will reduce the information sharing willingness of supply chain participants, so it is necessary to formulate constraint mechanism and confidentiality agreement to reduce the risk of information leakage.

Although some useful conclusions and management implications have been obtained in this article, there is still a lot of research space in the future. Further research can consider increasing government subsidy variables to explore the impact of subsidy mechanism on upstream and downstream enterprises' participation in information sharing.

\section{Data Availability}

The corresponding data used to support the findings of this study are included within the article.

\section{Conflicts of Interest}

The authors declare that they have no conflicts of interest.

\section{Acknowledgments}

This research was supported by the National Natural Science Foundation of China (Grant no. 71471073) and Fundamental Research Funds for the Central Universities (Grant no. CCNU19TS078).

\section{References}

[1] C. Mena, L. A. Terry, A. Williams, and L. Ellram, "Causes of waste across multi-tier supply networks: cases in the UK food sector," International Journal of Production Economics, vol. 152, no. 6, pp. 144-158, 2014.

[2] Y. Yang, T.-J. Fan, and L. Zhang, "Coordination of fresh agricultural supply chain with asymmetric freshness information," Chinese Journal of Management Science, vol. 24, no. 9, pp. 147-155, 2016, in Chinese.

[3] M. E. Porter, "Competitive strategy," Journal of Marketing, vol. 1, no. 2, 1980.

[4] H. Fu and M. Jian, "Supply chain information management strategy under information leakage," Computer Integrated Manufacturing Systems, vol. 20, no. 8, pp. 2170-2178, 2015, in Chinese.

[5] F. W. Harris, "How many parts to make at once," Operations Research, vol. 38, no. 6, pp. 947-950, 1990.

[6] P. L. Abad, "Optimal price and order size for a reseller under partial backordering," Computers \& Operations Research, vol. 28, no. 1, pp. 53-65, 2001.

[7] S. F. Schrader, "A model for exponentially decaying inventory," The Journal of Industrial Engineering, vol. 14, pp. 238-243, 1963.

[8] H. L. Lee, V. Padmanabhan, and S. Whang, "Information distortion in a supply chain: the bullwhip effect," Management Science, vol. 43, no. 4, pp. 546-558, 1997. 
[9] G. P. Cachon and M. Fisher, "Supply chain inventory management and the value of shared information," Management Science, vol. 46, no. 8, pp. 1032-1048, 2000.

[10] H. Ding, B. Guo, and Z. Liu, "Information sharing and profit allotment based on supply chain cooperation," International Journal of Production Economics, vol. 133, no. 1, pp. 70-79, 2011.

[11] L. Jraisat, M. Gotsi, and M. Bourlakis, "Drivers of information sharing and export performance in the Jordanian agri-food export supply chain," International Marketing Review, vol. 30, no. 4, pp. 323-356, 2013.

[12] F. Ye, X.-M. Chen, and Q. Lin, “Analysis of supply chain's demand information sharing values based on decisionmaker's risk aversion characteristics," Journal of Industrial Engineering and Engineering Management, vol. 26, no. 3, pp. 176-196, 2012, in Chinese.

[13] L. Guo, T. Li, and H. Zhang, "Strategic information sharing in competing channels," Production and Operations Management, vol. 23, no. 10, pp. 1719-1731, 2015.

[14] Y. Wang, "Game and incentives in the information sharing among supply chain members," Chinese Journal of Management Science, vol. 13, no. 5, pp. 61-66, 2005, in Chinese.

[15] M. Esmaeili and P. Zeephongsekul, "Seller-buyer models of supply chain management with an asymmetric information structure," International Journal of Production Economics, vol. 123, no. 1, pp. 146-154, 2010.

[16] J. M. Smith, "The theory of games and the evolution of animal conflict," Journal of Theory Biology, vol. 47, no. 1, pp. 209-221, 1973.

[17] J. M. Smith and G. R. Price, "The logic of animal conflicts," Nature, vol. 246, no. 1, pp. 15-18, 1974.

[18] L. A. Bach, T. Helvikc, and F. B. Christiansen, "The evolution of n-player cooperation-threshold games and ESS bifurcations," Journal of Theoretical Biology, vol. 238, no. 1, pp. 426-434, 2006.

[19] D. K. Levine and W. Pesendorfer, "The evolution of cooperation through imitation," Games and Economic Behavior, vol. 58, no. 2, pp. 293-315, 2007.

[20] X. Cai, J. Chen, Y. Xiao, X. Xu, and G. Yu, "Fresh-product supply chain management with logistics outsourcing," Omega, vol. 41, no. 4, pp. 752-765, 2013.

[21] B. H. Guo, Z. G. Fang, and Q. Liu, "“Study of regional energyintensive industry's exit mechanism based on evolutionary game," Chinese Journal of Management Science, vol. 20, no. 4, pp. 79-85, 2012, in Chinese.

[22] B. Dan and J. Chen, "Coordinating Fresh Agricultural Supply Chain under the Valuable Loss," Chinese Journal of Management Science, vol. 16, no. 5, pp. 42-49, 2008, in Chinese. 